Check for updates

Cite this: RSC Adv., 2017, 7, 53445

Received 20th September 2017 Accepted 6th November 2017

DOI: 10.1039/c7ra10436g

rsc.li/rsc-advances

\title{
KISS1 gene suppresses metastasis of
nasopharyngeal cancer via activation of the ERK1/2 \\ KISS1 gene suppresses metastasis of
nasopharyngeal cancer via activation of the ERK1/2 pathway
}

\author{
Tingting Li, ${ }^{a}$ Qian Sun, ${ }^{a}$ Yan Zhou, ${ }^{a}$ Zelai He, ${ }^{a}$ Hao Liu, ${ }^{b}$ Ping Xiang, ${ }^{c}$ Jin Xi, ${ }^{d}$ \\ Xiazi Zhang ${ }^{d}$ and Hao Jiang (D)*a
}

\begin{abstract}
Nasopharyngeal cancer (NPC) is one of the most common head and neck cancers in the world. The failure of the treatment of NPC is mainly caused by the metastasis of the cancer cells. There is a great need to study the mechanism of metastasis for accurate diagnosis, prognosis and efficient therapeutic strategy. The KISS1 gene has already been reported as a metastasis suppressor in numerous cancers. In this study, by integrating the Transwell assay, western blotting and real-time reverse transcriptase polymerase chain reaction (RTPCR) analysis, we found that the expression of KISS1 and its receptor gene (hOT7T175, KISS1R) negatively related with the metastasis of NPC cells. Overexpression of the KISS1 and KISS1R genes reduced migration and invasion of the SUNE-1-5-8F cells that are a cell line of NPC. Additionally, overexpression of these genes significantly increased the phosphorylation of focal adhesion kinase (FAK) and decreased the expression of ezrin (EZR), indicated by western blotting. Further study showed the metastasis suppression role of KISS1 and KISS1R was mediated though phosphorylation of the ERK1/2 pathway for that blocking the phosphorylation of the pathway with inhibitor (PD98059) reversed the metastasis suppression and phosphorylation of FAK as well as up-regulation of EZR simultaneously. Together, all of these results suggested for the first time that KISS1 and KISS1R suppress the metastasis of NPC cells by phosphorylation of FAK and decreasing EZR through phosphorylation of the ERK1/2 pathway.
\end{abstract}

\section{Introduction}

Nasopharyngeal cancer (NPC), as one of the most common head and neck cancers, ${ }^{\mathbf{1}}$ is vastly more common in certain regions of East Asia and Africa than elsewhere around the world. ${ }^{2,3}$ According to previous reports, about $80 \%$ percent of the NPC cases occur in Asia and 50000 deaths occur from it annually. ${ }^{4}$ Based on the characteristics, NPC cases are mainly divided into three groups: keratinizing squamous cell carcinoma, differentiated cell carcinoma and undifferentiated non-keratinizing cell carcinoma. ${ }^{5}$ In clinical practice, the most effective treatment for NPC has been a combination of radiotherapy and chemotherapy. Cisplatin and 5-fluorouracil are frequently used as chemotherapeutics. ${ }^{6,7}$ At one year, the survival rate with radiochemotherapy is $100 \%$, while at three years and at five years it is $94 \%$ and $73 \%$ respectively. ${ }^{8}$ The failure of the treatment is

${ }^{a}$ Department of Radiation Oncology, The First Affiliated Hospital of Bengbu Medical College, 287 Changhuai Road, Bengbu, Anhui 233004, R. P. China. E-mail: jianghao1223@163.com

${ }^{b}$ Laboratory of Pharmacology of Bengbu Medical College, 2600 Donghai Avenue, Bengbu, Anhui 233030, R. P. China

${ }^{c}$ Central Laboratory of the First Affiliated Hospital of Bengbu Medical College, 287 Changhuai Road, Bengbu, Anhui 233004, R. P. China

${ }^{d}$ Anhui Key Laboratory of Tissue Transplantation, Bengbu Medical College, 2600 Donghai Avenue, Bengbu, Anhui 233030, R. P. China mainly caused by surgery failure and the metastasis of cancer cells. Considering the side effects and high rates of failure with the conventional treatment strategies, there is a great need to develop new therapeutic approaches or medicines. The suppression of metastasis is an ideal candidate target for treatment.

Numerous studies have found that tobacco smoke, EpsteinBarr virus, nitrosamines consumption, genetic predisposition and racial patterns were associated with the development of NPC. ${ }^{9,10}$ Also, molecular substrates, such as Major Histocompatibility Complex Class I, A2, B14 and B16 play important role in the development of NPC. Additionally, cell adhesion/ migration genes like matrix metalloproteinase 2 (MMP2) and ezrin (EZR), cell cycle control genes like mouse double minute 2 human homolog (MDM2) and tumor protein p53 (TP53), as well as the ERBB-PI3K pathway and autophagy activation are reported regulating the development of NPC. ${ }^{\mathbf{1 1}, 12}$ However, the mechanism underlying the metastasis of NPC cells has not been clearly elucidated.

The KISS1 gene was first described by Lee et al., ${ }^{13}$ works as a metastasis suppressor gene in metastatic melanoma. KISS1 encodes a 145 amino acids protein, which is then cleaved into various peptides, namely kisspeptin-10, kisspeptin-13, kisspeptin-14 and kisspeptin-54 that ultimately bind to the G protein-coupled receptor. ${ }^{\mathbf{1 4 , 1 5}}$ It is well established that KISS1 
and $K I S S 1 R$ can suppress the metastasis of various cancer cells, such as melanoma, breast cancer, ${ }^{\mathbf{1 6}}$ gastric cancer, ${ }^{17}$ esophageal carcinoma $^{18}$ and pancreatic cancer ${ }^{19}$ and the loss of KISS1 and $K I S S 1 R$ expression also has been reported..$^{18}$ In addition, it has been reported that the phosphorylation of the ERK1/2 pathway and phosphorylation of the FAK, formation of stress fibers and focal adhesion were also induced after expression of the KISS1 gene, which subsequently led to the suppression of the migration and invasion of cancer cells. ${ }^{20}$ Additionally, overexpression of KISS1 was reported to decrease the expression of matrix metalloproteinase 9 (MMP9) and subsequently reduced the invasion. ${ }^{21}$ However, the roles of KISS1 and KISS1R during the development of NPC has not been reported to date. Based on previous studies, we want to determine the mechanism of metastasis suppress function of KISS1 and KISS1R in this study. Using RT-PCR and western blot, we found that the expression of KISS1 and KISS1R are negatively related with metastasis of NPC cells. In addition, using the Transwell assay, we found that overexpression of KISS1 and KISS1R, separately or together, significantly suppressed the migration and invasion of NPC cells. Moreover, the phosphorylation of the FAK was increased, while the actin-binding protein EZR was decreased. Further study showed the ERK1/2 pathway was activated though phosphorylation. Additionally, blocking the phosphorylation of this pathway with inhibitor (PD98059) reversed the suppression of the migration and invasion of the NPC cells, as well as the phosphorylation of the FAK and down-regulation of EZR caused by the overexpression of KISS1 and KISS1R.

\section{Materials and methods}

\section{Cell culture and chemicals}

The subtypes of the NPC cell line, namely SUNE-1-5-8F with high metastasis and SUNE-1-6-10B with low metastasis were purchased (Guangzhou Geneseed Biotech. Co., Guangzhou, China). The cells were cultured in Dulbecco's modified Eagle's medium (DMEM, Gibco, Grand Island, NY, USA), supplemented with $2 \mathrm{mM}$ L-glutamine, 10\% heat-inactivated fetal bovine serum (FBS) and antibiotics $\left(100 \mathrm{U} \mathrm{mL}^{-1}\right.$ penicillin and $100 \mu \mathrm{g}$ $\mathrm{mL}^{-1}$ streptomycin). The cells were cultured at a density of $5 \times$ $10^{4}$ cells per $\mathrm{mL}$ in an incubator with an atmosphere of $5 \% \mathrm{CO}_{2}$ and $95 \% \mathrm{O}_{2}$. The culture medium was exchanged every $2-3$ days and cells were sub-cultured twice a week. The antibodies for KISS1, ERK1/2, FAK, phosphorylation of FAK (tyr397) and EZR were purchased from Santa Cruz Biotechnology (Santa Cruz, CA, USA). The inhibitor of the ERK1/2 pathway (PD98059) was purchased from Sigma (Saint Louis, MO, USA).

\section{Overexpression of the KISS1 and KISS1R genes in SUNE-1-5- $8 \mathrm{~F}$ cells}

The cell line that stably expressing the KISS1R gene was constructed with the pSIREN-RetroQ retroviral vector (Clontech, Mountain View, CA, USA). First, the pSIREN-RetroQ retroviral vector containing the complementary DNA (cDNA) encoding the polypeptide of the KISS1R and the vectors encoding GFP alone were used to transfect $293 \mathrm{~T}$ cells and the supernatant was collected $48 \mathrm{~h}$ later. The supernatants were applied to SUNE-5$8 \mathrm{~F}$ cells with $5 \mu \mathrm{g} \mathrm{mL} \mathrm{m}^{-1}$ polybrene followed by the addition of $1 \mu \mathrm{g} \mathrm{mL}{ }^{-1}$ puromycin $24 \mathrm{~h}$ later for selection of the infected cells (referred as SUNE-1R and SUNE-GFP cells). Subsequently, the cells were selected and proliferated. For the overexpression of KISS1, $4 \mu \mathrm{g}$ of the plasmid containing the KISS1 cDNA sequence or a negative control were mixed with $8 \mu \mathrm{g}$ Lipofectamine 2000 (Invitrogen, Carlsbad, CA, USA) and used to transfect the SUNE-1R cells or the SUNE-GFP cells for $24 \mathrm{~h}$ according to the manufacturer's instructions. Each experiment was replicated four times. After $24 \mathrm{~h}$ of the transfection, the cells were sampled for the experiments.

\section{KISS-1 interference with siRNA}

The specific siRNA (5'-GCCGAACUACAACUGGA ACTT- $\left.3^{\prime}\right)$ with GFP tags for KISS-1 gene and negative control (NC) that constructed in lentivirus were bought (Genechem Biotech. Co., Shanghai, China). The SUNE-1-5-8F cells were transfected according to the instruction. Briefly, the cells were adjusted to 1 $\times 10^{4}$ cells per $\mathrm{mL}$ in one of 24 -well plate before transfection. The most effective lentivirus $\left(10 \mu \mathrm{L}, 2 \times 10^{7} \mathrm{TU} \mathrm{mL}^{-1}\right.$ and $90 \mu \mathrm{L}$ medium) determined by multiplicity of infection (MOI) was used to transfect the cells with Lipofectamine 2000 and then the transfected cells were selected with $50 \mu \mathrm{g} \mathrm{mL}{ }^{-1}$ puromycin. The metastasis (migration and invasion) and phosphorylation of ERK1/2 was detected $96 \mathrm{~h}$ after transfection.

\section{Real time polymerase chain reaction (PCR) analysis}

For the RT-PCR analysis, the total RNA was extracted from the cells using the TRIZOL (Sangon Biotech., Shanghai, China) reagent according to manufacturer's instructions. The purity and concentration of the isolated RNA was determined by UV spectrophotometry. Then, the cDNA was synthesized using a RT-PCR kit (Sangon Biotech.) and amplified in a reaction mixture containing $10 \mu \mathrm{L}$ of $2 \times$ SYSBR Green Master Mix, $0.5 \mu \mathrm{L}$ of each primer of KISS1 (forward: 5'-AGCCGCCAGATCCCCGCA$3^{\prime}$; reverse: $5^{\prime}$-GCCGAA GGAGTTCCAGTTGTAGTT- $3^{\prime}$ ), $1 \mu \mathrm{L}$ cDNA template, and $8 \mu \mathrm{L} \mathrm{ddH_{2 }} \mathrm{O}$. In this experiment, the GAPDH gene was used as reference. The relative gene expression was analyzed with the Data Assist software version 3.0 (Applied Biosystems, Foster City, CA, USA).

\section{Western blot analysis}

The proteins of interest in this study were analyzed by Western blot analysis. After transfection of SUNE-5-8F cells with KISS1 or negative control for $48 \mathrm{~h}$, the obtained SUNE-1R or SUNE-GFP cells, were washed 3 times with PBS and then lysed in $100 \mu \mathrm{L}$ RIPA buffer (Sangon Biotech.). Subsequently, the supernatant of the lysates were collected after centrifugation $(12000 \mathrm{~g})$ at $4{ }^{\circ} \mathrm{C}$ for $20 \mathrm{~min}$. The concentration of the protein samples were determined with the BCA assay kit (Beyotime, China), using GAPDH as reference. Afterwards, 20 $\mu \mathrm{g}$ of the prepared proteins were separated using $10 \%$ sodium dodecylsulfate polyacrylamide gel electrophoresis (SDSPAGE) and subsequently transferred onto polyvinylidene difluoride (PVDF; Millipore, Billerica, MA, USA) membranes 
at $15 \mathrm{~V}$ for $30 \mathrm{~min}$. The membranes were blocked with $5 \%$ non-fat milk for $1 \mathrm{~h}$ at room temperature and then washed three times for 5 min with TBST. The membranes were incubated with the corresponding primary antibodies at room temperature for $1 \mathrm{~h}$ and subsequently incubated with the appropriate secondary HRP antibodies for $40 \mathrm{~min}$. Finally, the membranes were washed three times with TBST before detection of the bands using a Gel Imaging System (Fusion Solo, VILBER, France).

\section{Migration and invasion analysis by Transwell assay}

The migration and invasion of SUNE-1-5-8F cells overexpressing $K I S S 1$ and its receptor KISS1R or not were analyzed using the Transwell assay (Corning Inc., Corning, NY, USA). Briefly, after expression of the transfected genes for $24 \mathrm{~h}$, the cells were washed twice with PBS and detached by digestion with $0.25 \%$ trypsin. Then, the cells were centrifuged for $5 \mathrm{~min}$ and re-suspended at 1 $\times 10^{5}$ cells per $\mathrm{mL}$ using DMEM without serum. The Transwell membranes with $8 \mu \mathrm{m}$ pore size (Corning Inc.) were placed in the wells of the 24-well plates containing $600 \mu \mathrm{L}$ DMEM with $10 \%$ FBS. Next, the membranes were seeded with $1 \times 10^{5}$ cells in 200 $\mu \mathrm{L}$ culture medium and incubated for $24 \mathrm{~h}$ in $5 \% \mathrm{CO}_{2}$ incubator. Finally, after fixing the cells on the Transwell membranes with $4 \%$ polyformaldehyde, images were captured using a Leica DM2500 (Leica Microsystems GmbH, Wetzlar, Germany) microscope and the cells were counted using Image (NIH, Bethesda, MD, USA). For the invasion assay, the polyethylene terephthalate membranes were coated with Matrigel (Sigma) and then the cells were seeded on the Matrigel. After culturing for $24 \mathrm{~h}$, the cells attached to membrane were fixed and counted.

\section{Wound healing assay}

For the wound healing assay, the cells were seeded on the sixwell plates with $1 \times 10^{5}$ cells per well and allowed to grow to a confluent monolayer in the incubator at $37{ }^{\circ} \mathrm{C}$ before wounding the monolayer by scratching the surface with $1 \mathrm{~mL}$ pipette tip. Then, the cells were washed for $3 \times 5 \mathrm{~min}$ and incubated for $24 \mathrm{~h}$, followed by fixing with $4 \%$ paraformaldehyde dissolved in phosphate buffer. The images were captured with a Leica DM2500 (Leica Microsystems GmbH) microscope.

\section{Statistical analysis}

All the data in this study are presented as the mean \pm SEM. The unpaired Student's $t$-test or ANOVA (one-way or two-way) test was used to statistically analyse the experimental data between different groups, to determine if differences among groups are significant. All the analyses were performed using the SPSS version 19.0 (IBM Corp., Armonk, NY, USA) or GraphPad Prism 6 (GraphPad Inc., La Jolla, CA, USA). $P$ values $<0.05$ was considered statistically significant.

\section{Results}

\section{KISS1 is highly expressed in SUNE-1-6-10B cells and lowly expressed in SUNE-1-5-8F cells}

First, we determined whether the metastasis of human NPC cells was associated with the expression of KISS1. The protein and mRNA expression of KISS1 were analysed in SUNE-1-6-10B and SUNE-1-5-8F cells which are poorly metastatic and highly metastatic, respectively. The RT-PCR expression analysis revealed that the mRNA of KISS1 is highly expressed in the SUNE-1-6-10B cells compared with the SUNE-1-5-8F cells (Fig. 1A). The Western blot protein expression analysis revealed that $K I S S 1$ and its receptor $K I S S 1 R$ were also highly expressed in SUNE-1-6-10B cells compared with SUNE-1-5-8F cells (Fig. 1B and $\mathrm{C}$ ), which is consistent with the RT-PCR analysis results. Meanwhile, the expression of the metastasis related phosphorylation of FAK was lower in SUNE-5-8F cells than in SUNE1-6-10B cells and the EZR expression was the opposite. While the expression of FAK was unchanged (Fig. 1B and C). According to these data, we can surmise that the expression of KISS1 was negatively associated with the metastasis of human NPC cells.

\section{Overexpression of KISS1 inhibited the migration of SUNE-1-5- $8 F$ cells}

To further evaluate of the role of KISS1 and KISS1R in the metastasis of human NPC cells separately or together, these genes were overexpressed in SUNE-1-5-8F cells. The SUNE-1R and SUNE-GFP cell lines that stably express the KISS1R or GFP alone in the SUNE-5-8F cells were generated (Fig. 2A and B). Then, the cells were transfected with the plasmid expressing the KISS1 gene. Compared with the SUNE-GFP cells expressing GFP

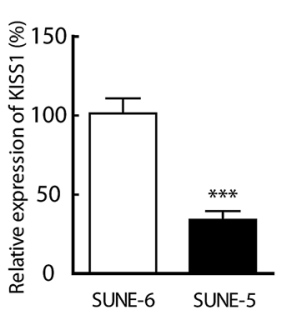

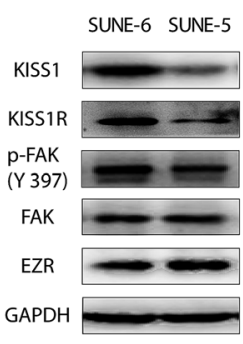

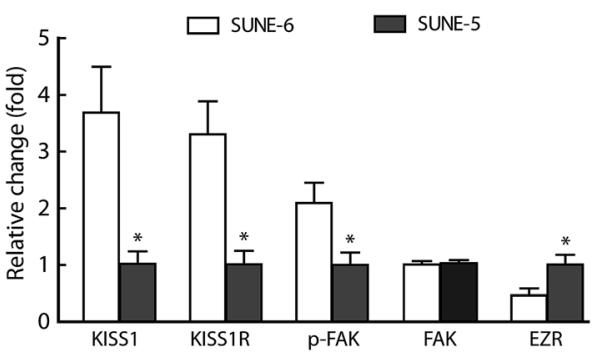

Fig. 1 Expression of the KISS1 gene negatively associated with metastasis of human nasopharyngeal cancer cells. (A) RT-PCR analysis shows relative expression of KISS1 in SUNE-1-5-8F compared with SUNE-1-6-10B. (B) Representative bands of KISS1R, KISS1, phosphorylated FAK ( $p$ FAK), FAK and EZR expressed in SUNE-1-6-10B and SUNE-1-5-8F cells. (C) Statistical data of the relative expression of KISSR1, KISS1, p-FAK, FAK and EZR expressed in SUNE-1-6-10B and SUNE-1-5-8F. Unpaired $t$ test. Data are presented as the mean \pm SEM, $* P<0.05, * * * P<0.001$. 
alone, the migration of the SUNE-1R was slightly inhibited as measured by the wound healing assay (Fig. 2C and D). In addition, the migration was significantly inhibited after the KISS1 gene was expressed in the SUNE-GFP (SUNE-KISS1) or in SUNE-1R (SUNE-1R-KISS1) (Fig. 2C and D). We also utilized the Transwell assay to analyze the migration of the cells. The results of the Transwell assay, in line with the wound healing assay, revealed that the migration was slightly and significantly suppressed after the receptor KISS1R and KISS1 were overexpressed separately (Fig. 3A and C). Furthermore, the migration was inhibited more intensely when KISS1 and KISS1R were both expressed simultaneously, but no statistical difference was detected when compared with expression of KISS1 alone (Fig. 3A and $\mathrm{C}$ ).

\section{Overexpression of KISS1 inhibited the invasion of SUNE-1-5- 8F cells}

For further evaluation of the metastasis suppression function of $K I S S 1$ and KISS1R, the impact on invasion were assayed with the
Transwell assay. The invasion was slightly and intensely suppressed in SUNE-1R and SUNE-KISS1 cells respectively (Fig. 3B and $\mathrm{D}$ ). The invasion was greatly inhibited when KISS1 and $K I S S 1 R$ were simultaneously overexpressed, however no significant statistical difference was observed when compared with the invasion of SUNE-KISS1 cells expressing the KISS1 gene alone (Fig. 3B and D).

\section{Overexpression of KISS1 increases the p-FAK and decreases expression of EZR}

To further investigate the underlying mechanism, the p-FAK and expression of EZR was analysed for that the functions of these protein in migration and invasion have been reported. ${ }^{\mathbf{1 4 , 2 2 , 2 3}}$ The KISS1 and KISS1R were successfully expressed in SUNE-1-5-8F cells as indicated by the significantly increased expression of KISS1R in SUNE-1R and KISS1 in SUNE-KISS1 cells (Fig. 4A-C). In addition, KISS1 was up-regulated after $K I S S 1 R$ was overexpressed (Fig. 4C). We also evaluated the expression of EZR and phosphorylation of FAK (tyr 397) after the
A
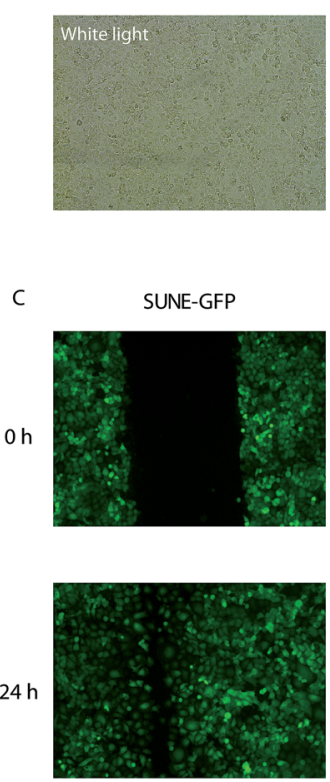

SUNE-GFP

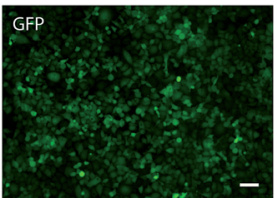

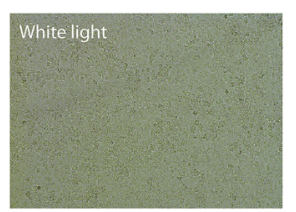

SUNE-KISS1
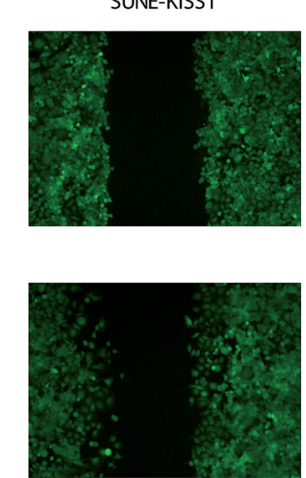

SUNE-1R

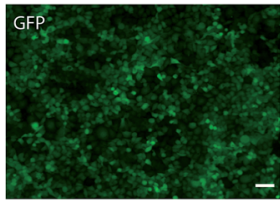

SUNE-1R-KISS1
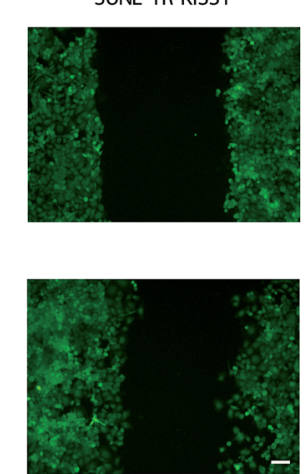

D
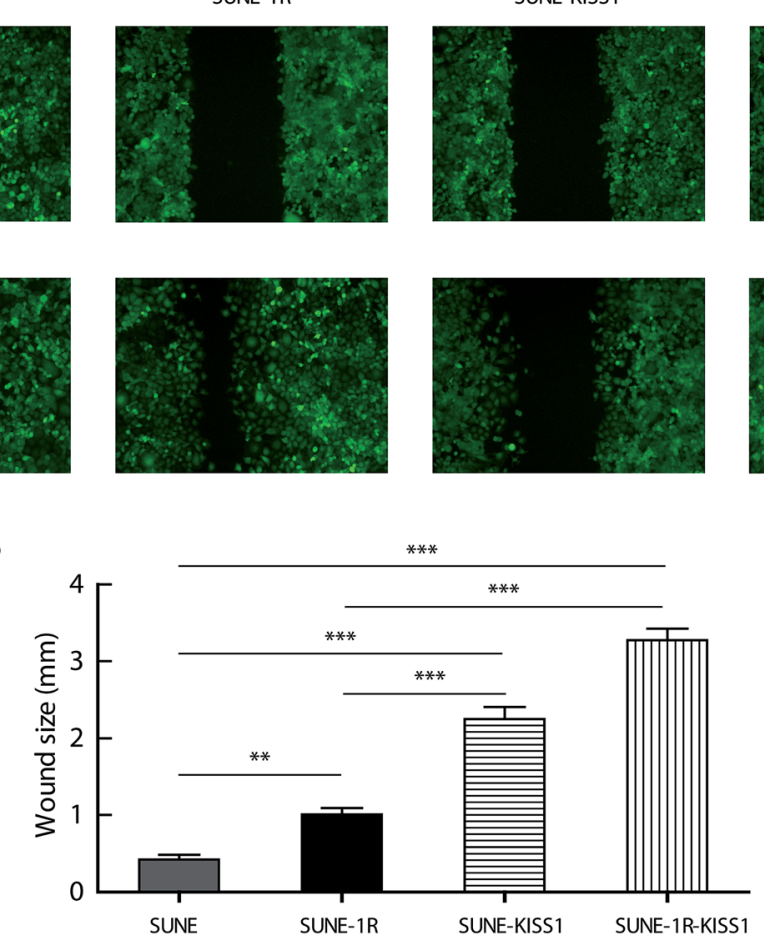

Fig. 2 Expression of KISS1 inhibited the migration of NPC cells detected by wound healing assay. (A and B) Typical images of GFP alone (A) and KISS1R-GFP (B) stably expressed in SUNE-1-5-8F cells. Scale bar, $500 \mu \mathrm{m}$. (C) Representative images of the wound healing assay of indicated groups (SUNE-GFP, SUNE-1R, SUNE-KISS1, SUNE-1R-KISS1) after KISS1 and KISS1R expressed together or separately for $24 \mathrm{~h}$. (D) Statistical data of the wound healing assay. Data are presented as the mean $\pm \mathrm{SEM}, * * P<0.01, * * * P<0.001$. 
A

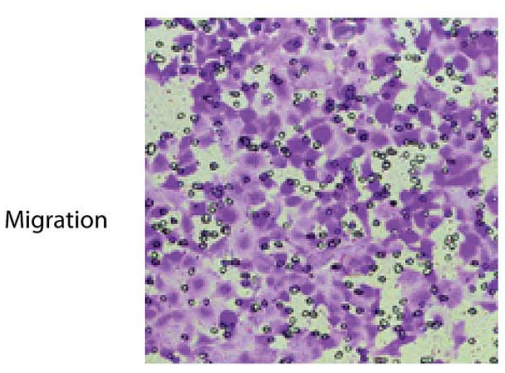

SUNE-1R

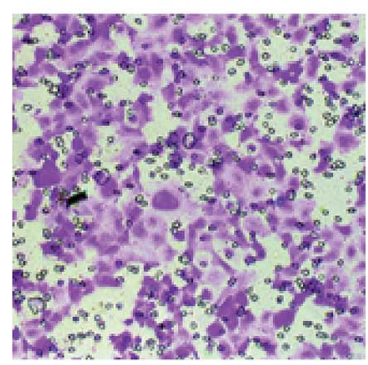

SUNE-KISS1

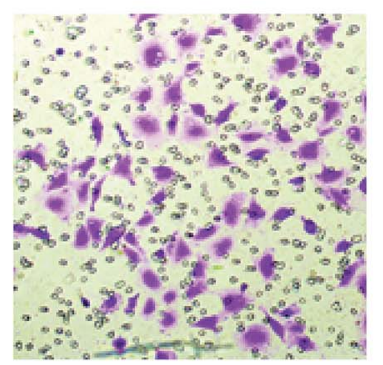

SUNE-1R-KISS1

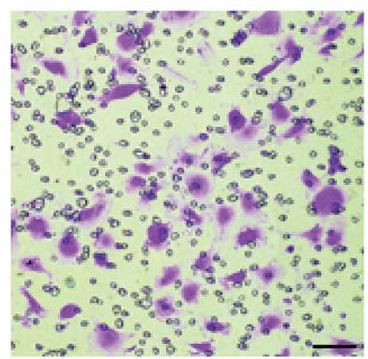

B

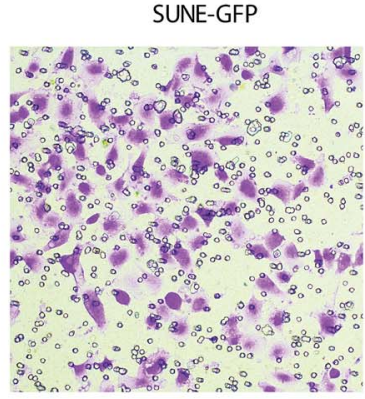

SUNE-1R

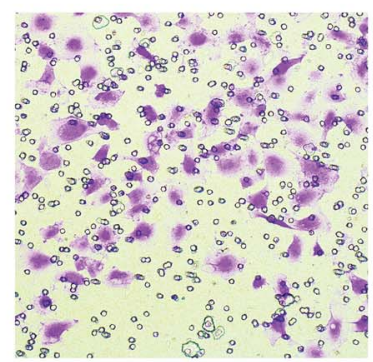

SUNE-KISS

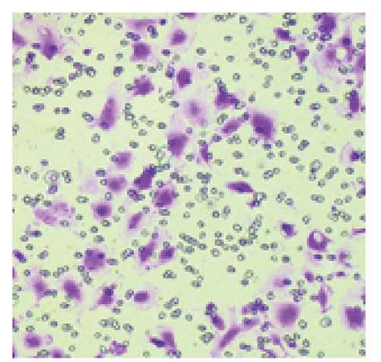

SUNE-1R-KISS1

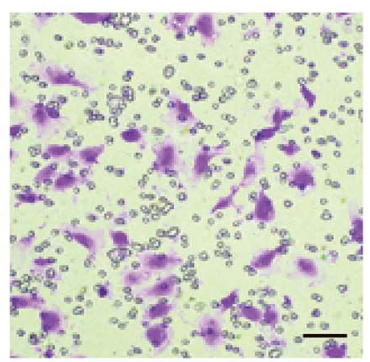

c

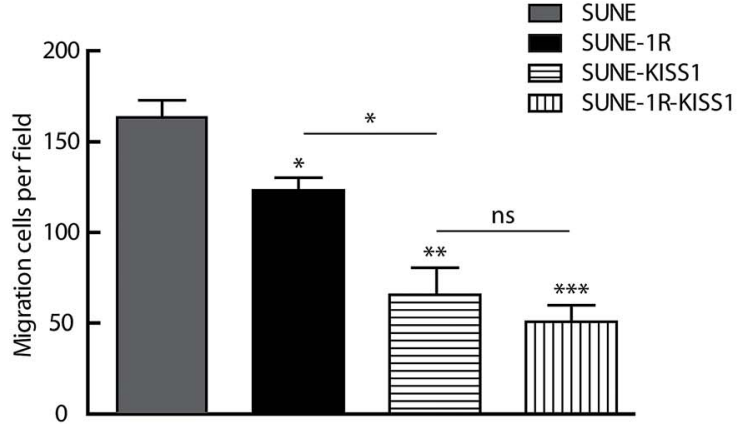

D

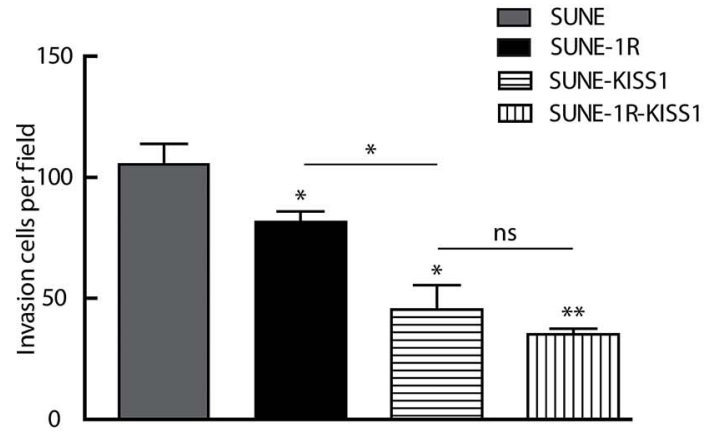

Fig. 3 Overexpression of KISS1 suppressed the migration and invasion of NPC cells. (A and B) Images of the Transwell assay showed the migration and invasion of indicated groups (SUNE-GFP, SUNE-1R, SUNE-KISS1, SUNE-1R-KISS1). Scale bar $50 \mu \mathrm{m}$. (C and D) Summary data of the migration (C) and invasion (D) of different groups (SUNE-GFP, SUNE-1R, SUNE-KISS1, SUNE-1R-KISS1) that expressed KISS1 and KISS1R separately or together. One-way ANOVA test. Data are presented as the mean $\pm \mathrm{SEM}$. $* P<0.05 ; * * P<0.01$.

overexpression of KISS1 and KISS1R alone or together. We found that the phosphorylation of FAK was significantly up-regulated, while the expression of EZR was significantly down-regulated when KISS1 and KISS1R were expressed alone or together compared with SUNE-GFP cells (Fig. 4A, D and E). No significant statistical difference between the SUNE-1R-KISS1 cells and the SUNE-KISS1 cells was detected.

\section{KISS1 increased phosphorylation of ERK1/2}

To determine how the expression of EZR and phosphorylation of FAK were regulated, the phosphorylation of the ERK1/2 pathway was assayed for the activation of the pathway was described in breast carcinoma and papillary thyroid cancer. ${ }^{\mathbf{2 0 , 2 4}}$ After overexpression of KISS1 and KISS1R, separately or together, the phosphorylation of the ERK1/2 was variously increased (Fig. 4F and G).
Inhibition of the ERK1/2 pathway reversed the metastasis suppression caused by expression of KISS1

In order to determine whether the phosphorylation of ERK1/2 pathway was necessary and sufficient for the metastasis suppression of KISS1 and KISS1R, the pathway was blocked with the inhibitor PD98059 $(25 \mu \mathrm{M})$. We found the migration and invasion suppression induced by overexpression of KISS1 and KISS1R were reversed after PD98059 was added to the cells for $24 \mathrm{~h}$ during the Transwell assay (Fig. 5A-D). The phosphorylation of the ERK1/2 pathway in the SUNE-1R and SUNE-1R-KISS1 cells was decreased (Fig. 6A and B). The Western blot analysis results showed that the phosphorylation of FAK and the downregulation of EZR were also reversed (Fig. 6C and D). Taking all these data together, we can conclude that KISS1 and its receptor $K I S S 1 R$ gene suppress metastasis of NPC cells by phosphorylation of FAK and the down-regulation of EZR through phosphorylation of the ERK1/2 pathway. 


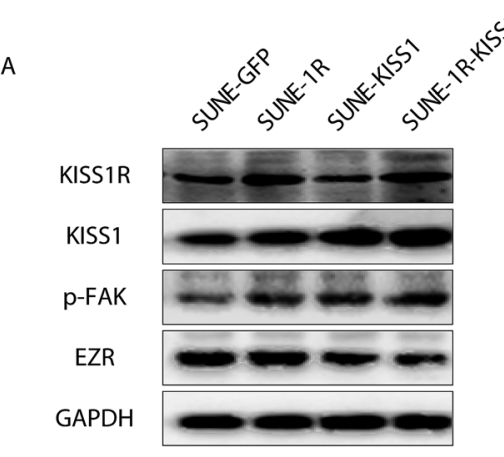

$B$
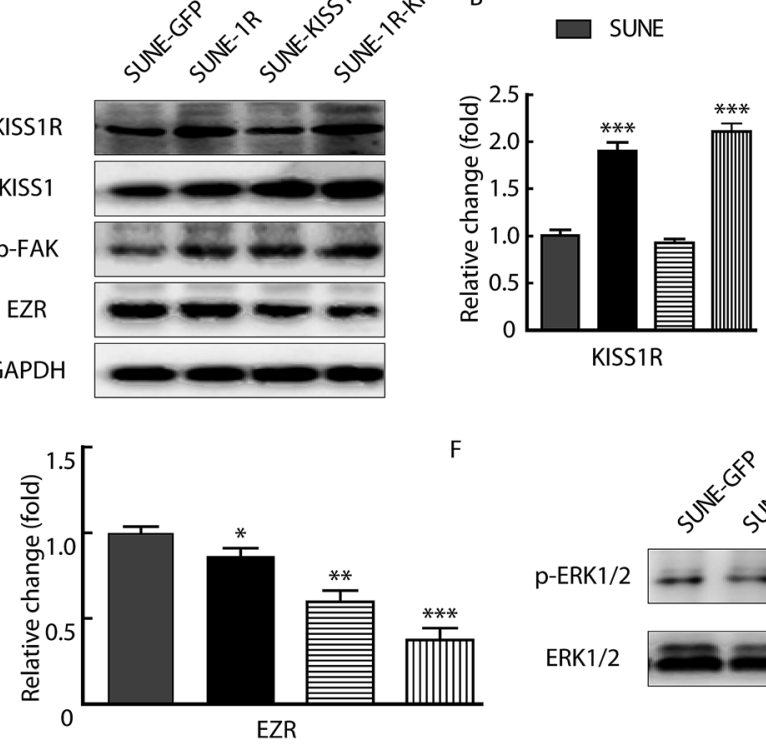

C

SUNE-1R $\quad$ E SUNE-KISS1

D
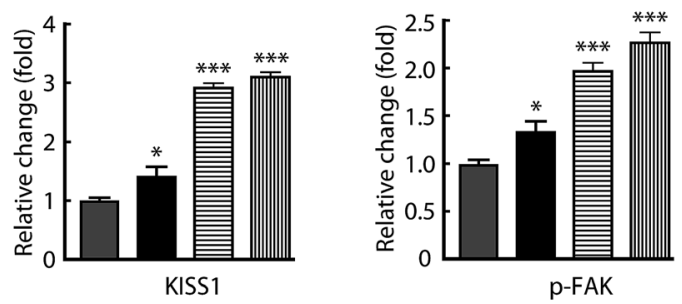

Fig. 4 Overexpression of KISS1 increased phosphorylation of FAK, ERK1/2 and decreased expression of EZR. (A) Representative bands that show the expression of KISS1R, KISS1, p-FAK, and EZR in SUNE, SUNE-1R, SUNE-KISS1 and SUNE-1R-KISS1 cells. (B-E) Statistical data of the expression of KISS1R, KISS1, p-FAK, FAK and EZR in indicated groups. (F) Bands of phosphorylated ERK1/2 (p-ERK1/2) in cells that overexpressed KISS1 and KISS1R separately or together. (G) Histogram showing that the phosphorylation of ERK1/2 pathway in indicated groups. Unpaired Student's $t$ test was used to analyse the difference between different groups. Data are presented as the mean $\pm \mathrm{SEM} . * P<0.05 ; * * P<0.01$.
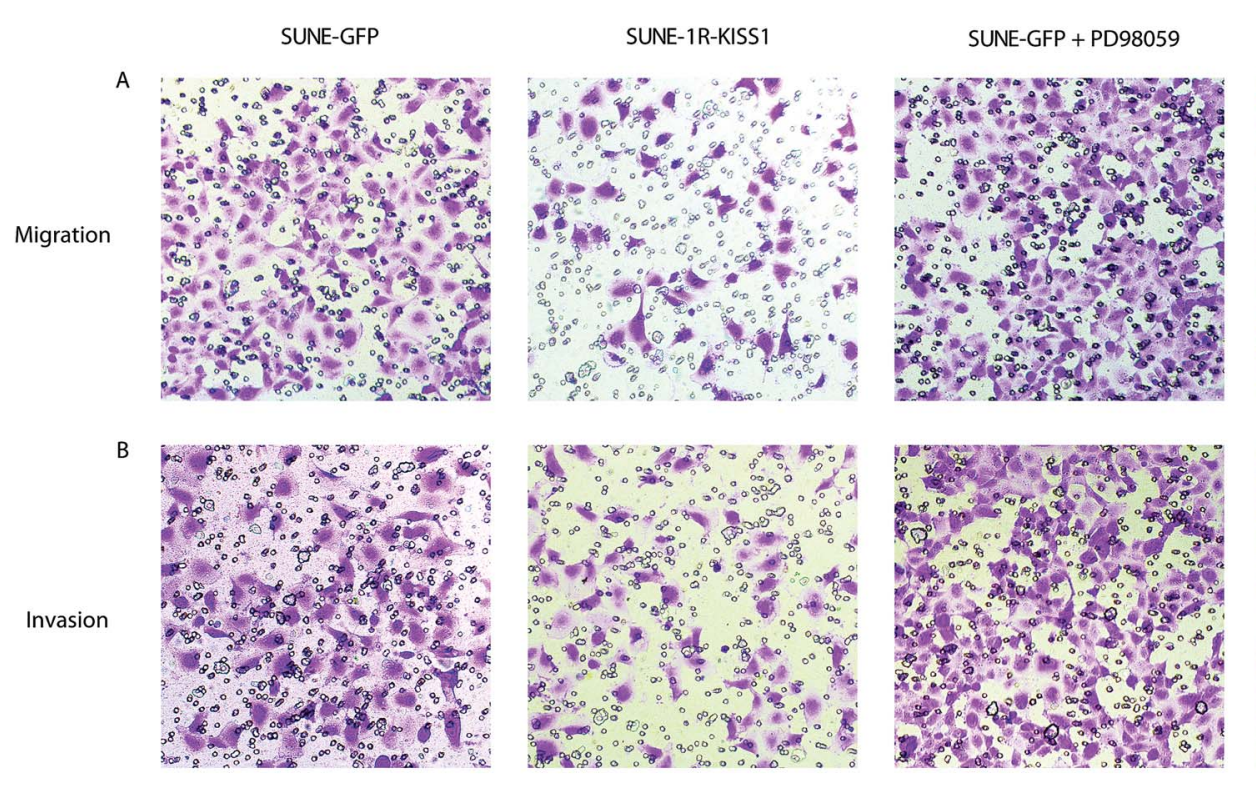

SUNE-1R-KISS1 +PD98059
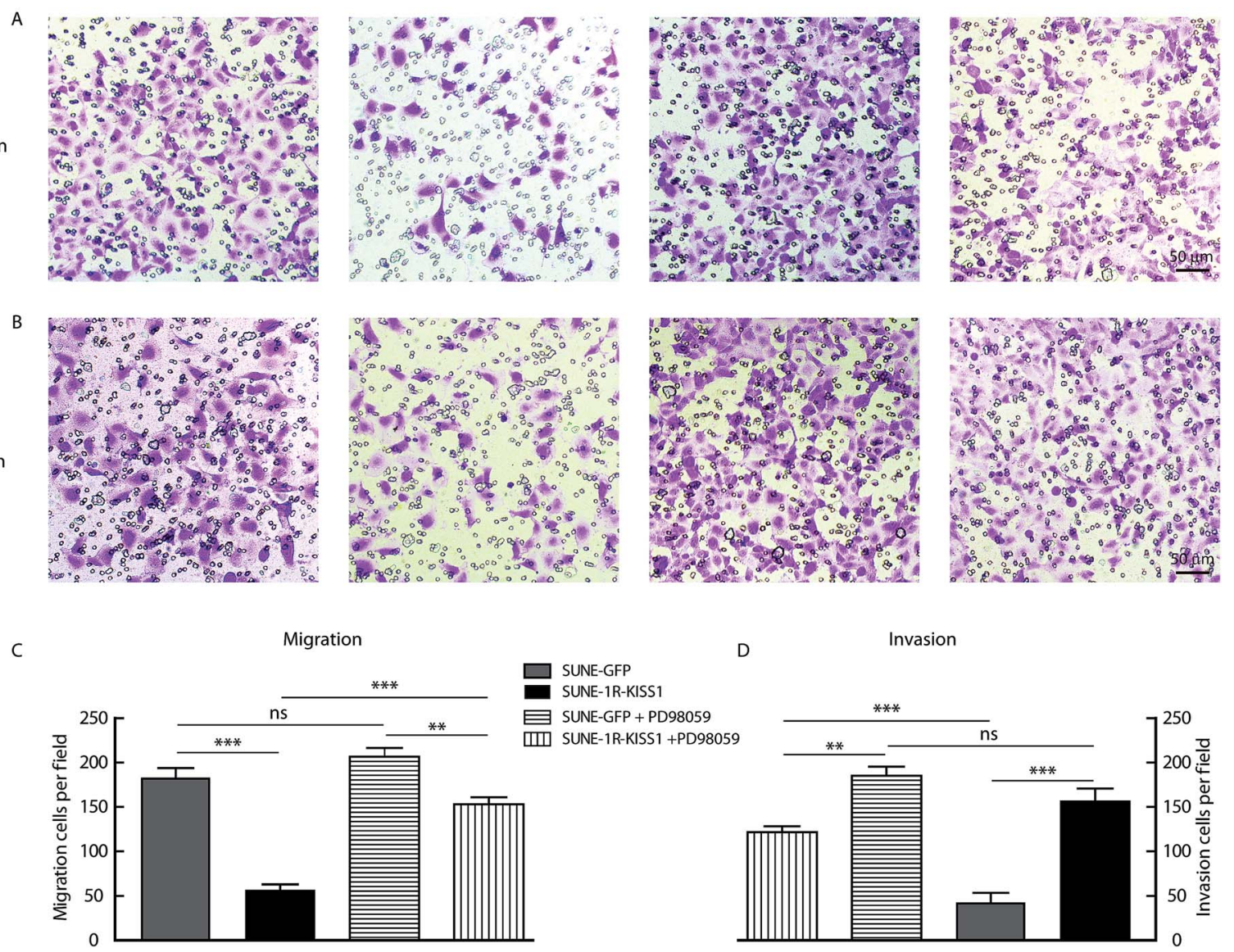

D

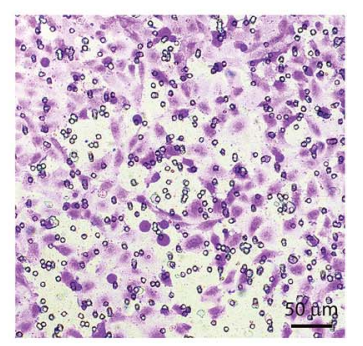

Invasion

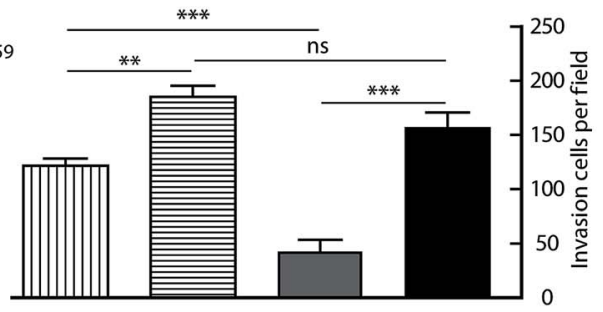

Fig. 5 Blocking the phosphorylation of ERK1/2 pathway reversed the suppressive effects induced by overexpression of KISS1 and KISS1R on the migration and invasion. (A and B) Images of the Transwell assay show that the migration (A) and invasion (B) of indicated groups with PD98059 or not. ( $C$ and D) Histogram shows summary data of the migration and invasion of indicated groups. Unpaired Student's $t$ test was used to analyze the difference between different groups. Data are presented as the mean $\pm \mathrm{SEM}$. $* P<0.05 ; * * P<0.01$. n.s. no significant. 
A

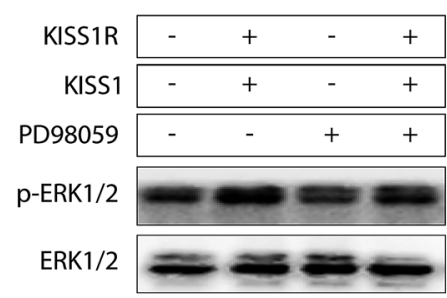

C

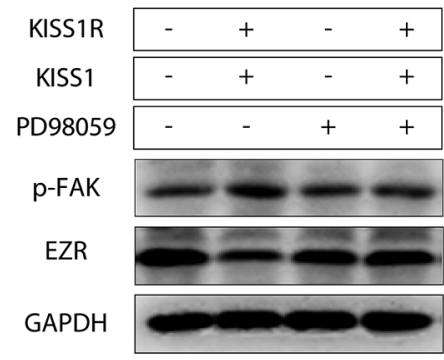

B

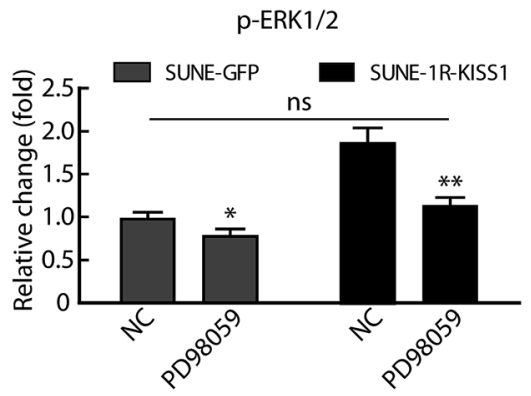

D

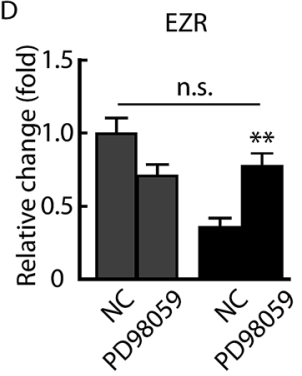

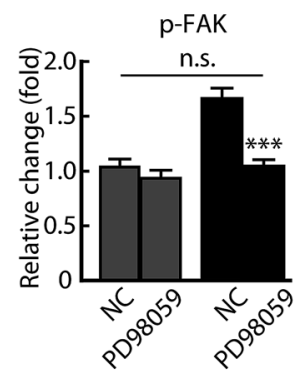

Fig. 6 Inhibition of the ERK1/2 pathway reversed the phosphorylation of the FAK, ERK1/2 and decrease of EZR that induced by overexpression of KISS1 and KISS1R. (A) Representative bands of the phosphorylation of ERK1/2 in indicated groups with PD98059 or not. (B) Summary data of the phosphorylation of the ERK1/2 pathway in indicated groups with PD98059 or not. NC, negative control. Unpaired Student's $t$ test. (C) Typical bands of p-FAK and EZR expression after overexpression of KISS1 and KISS1R in the presence or absence of PD98059. (D) Statistical data of EZR and $p$-FAK in indicated groups with PD98059 or not. Unpaired Student's $t$ test. Data are presented as the mean \pm SEM. $* P<0.05 ; * * P<0.01 ; * * * P$ $<0.001$. n.s. no significant.

Interference of KISS1 increased and metastasis of NPC by decreasing phosphorylation of ERK1/2 pathway

In order to validation the critical function of KISS1 in NPC, the KISS1 was suppressed with siRNA as previously reported. ${ }^{25}$ As showed in Fig. 7, the migration and invasion were significantly increased (Fig. 7B-E). Meanwhile, the phosphorylation of ERK1/ 2 pathway was significantly decreased (Fig. $7 F$ and G). All these data in line with the results of overexpression of KISS1 and clinical research. ${ }^{18}$

\section{Discussion}

In clinical practice, the invasion and metastasis of NPC cells represent a challenge for the treatment of this cancer and is the leading reason for the failure of the treatment and death. Accordingly, there is a great need to investigate the underlying mechanism of the metastasis of NPC cells for the accurate diagnosis, prognosis and development of efficient therapeutic strategy. Even though numerous factors have been reported regulating the development of NPC, the underlying mechanism remains unclear.

The invasion and metastasis of the cancer cells are lethal characteristics and mainly depend on the adhesion with surrounding tissues. Phosphorylation of the FAK has been found to participate in the regulation of cell migration, proliferation and survival. ${ }^{26,27}$ Additionally, it also plays a critical role in the metastasis of cancer cells. ${ }^{28-30}$ It is also well established that EZR, as a member of the ERM (ezrin-radixin-moesin) family of cytoskeletal proteins, also plays a crucial role in cell adhesion, migration and organization. Increased expression of EZR has been reported in various cancers, such as NPC, breast carcinoma and squamous cell carcinoma. ${ }^{31}$

KISS1 was first reported by Lee JH et al., in 1996 as a metastasis-suppressor gene in numerous cancers, such as colorectal cancer, melanoma, breast cancer, gastric cancer, esophageal carcinoma and pancreatic cancer. Besides, KISS1R was also reported to play an important role in esophageal squamous cell carcinoma. ${ }^{18}$ However, few studies have paid attention to the role of KISS1 and KISS1R in NPC metastasis and the underlying mechanism. In this study, the metastasis suppressor function of KISS1 and KISS1R in NPC cells metastasis is described for the first time.

In this study, using RT-QPCR and western blot analyses, we found that the expression of KISS1 and KISS1R were significantly increased in SUNE-1-6-10B cells that are less metastatic than SUNE-1-5-8F cells, which are highly metastatic. These data suggested that the metastasis of NPC cells may be negatively related with the expression of KISS1 and KISS1R. The negative relation of expression of KISS1 and KISS1R miRNA with cancers has already reported in pancreatic cancer. This means the RNA interference of KISS1 and KISS1R should increases the metastasis of cancer cells. Moreover, the migration and invasion of NPC cells were significantly suppressed after KISS1 and its receptor KISS1R gene overexpressed separately and together in UNE-1-5-8F cells. By Western blot analysis, we found that the phosphorylation of the FAK was significantly increased and the expression of EZR was dramatically decreased after overexpression of KISS1 and KISS1R. Additional phosphorylation 

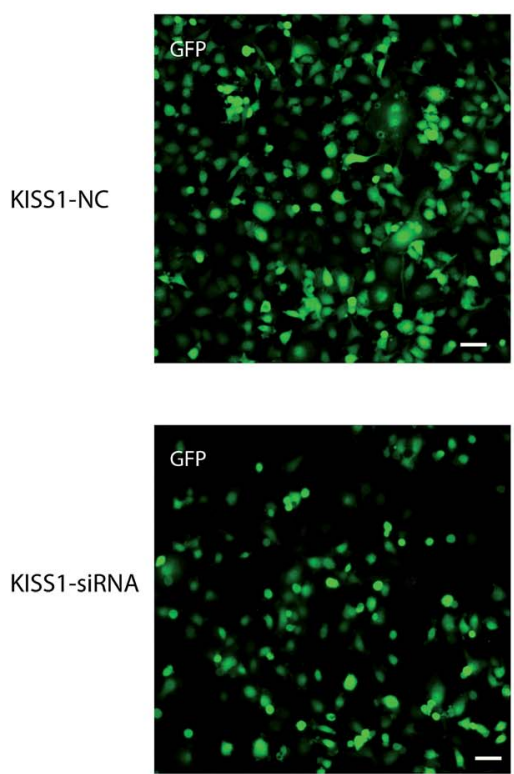

D

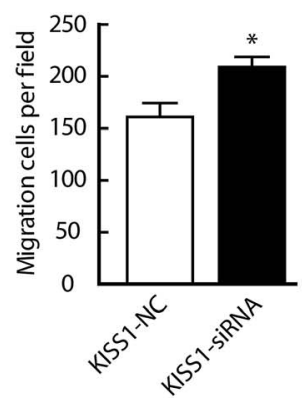

B
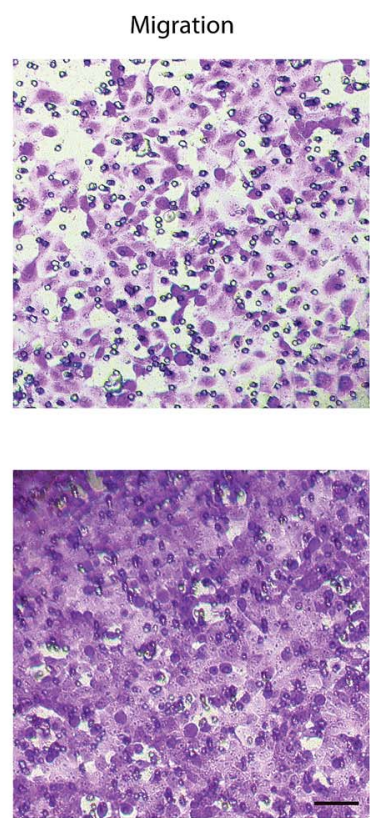

C
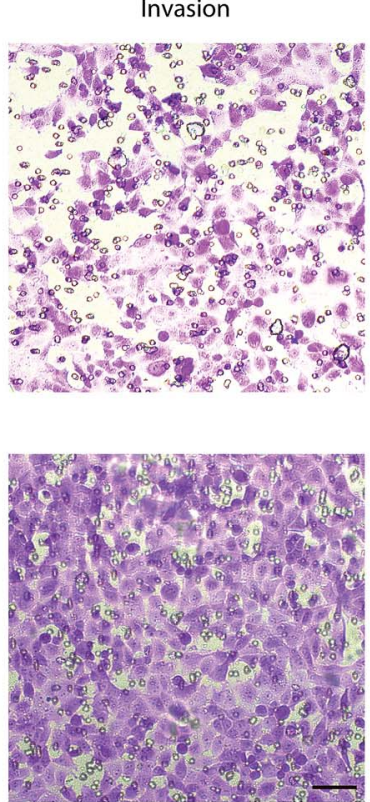

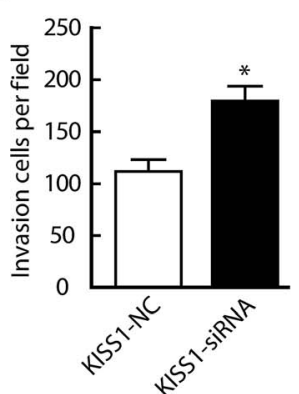

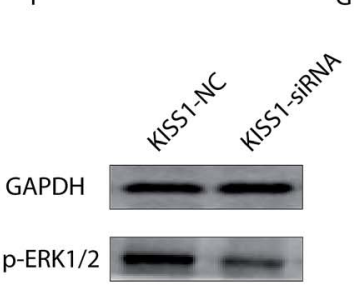

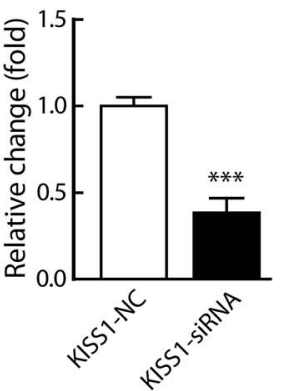

Fig. 7 Suppression of KISS1 increased and metastasis of NPC by decreasing phosphorylation of ERK1/2 pathway. (A) Representative images SUNE-1-5-8F that transfected with siRNA of KISS1 and negative control. Scale bar, $500 \mu \mathrm{m}$. (B and C) Representative images of migration (B) and invasion (C) of indicated groups. Scale bar, $50 \mu \mathrm{m}$. (D and E) Statistical data of migration (D) and invasion (E) of indicated groups. (F and G) Representative bands of phosphorylation of indicated groups (F) and the statistical data (G). Unpaired Student's $t$ test. Data are presented as the mean \pm SEM. $* P<0.05 ; * * * P<0.001$.

analysis studies further showed that the ERK1/2 pathway was phosphorylated and inhibition of the ERK1/2 pathway with the inhibitor reversed the changed expression of EZR and phosphorylation of the FAK caused by overexpression of KISS1. However, the statistical difference between metastasis suppression in SUNE-1R and SUNE-KISS1 cells (Fig. 3C and D), as well as the difference between SUNE-GFP and SUNE-1R-KISS1 after treatment with PD98059 (Fig. 5C and D) indicated that the KISS1 gene may work through another receptor or pathway in NPC cells. The critical function of the KISSS1 and KISS1R genes in NPC cells reported in this study provides a new insight regarding the underlying mechanism of the metastasis of NPC cells and reveals a new target for the development of a novel treatment strategy and for the diagnosis of NPC in clinical practice. According to clinical studies and our results in this study, we partly revealed the mechanism of occurrence of cancers and the expression of KISS1 and KISS1R could be an indicator for diagnosis.

\section{Conflicts of interest}

There are no conflicts to declare.

\section{Acknowledgements}

This work is supported by Anhui Provincial National Natural Science (1408085MH190).

\section{References}

1 V. T. DeVita, S. Hellman and S. A. Rosenberg, Cancer, principles \& practice of oncology, Lippincott Williams \& Wilkins, Philadelphia, PA, 7th edn, 2005.

2 N. Mahdavifar, M. Ghoncheh, A. Mohammadian-Hafshejani, B. Khosravi and H. Salehiniya, Osong Public Health and Research Perspectives, 2016, 7, 360-372.

3 G. Bar-Sela, A. Kuten, I. Minkov, E. Gov-Ari and O. Ben-Izhak, J. Clin. Pathol., 2004, 57, 290-293. 
4 D. M. Parkin, F. Bray, J. Ferlay and P. Pisani, Ca-Cancer J. Clin., 2005, 55, 74-108.

5 T. L. Vaughan, J. A. Shapiro, R. D. Burt, G. M. Swanson, M. Berwick, C. F. Lynch and J. L. Lyon, Cancer Epidemiol., Biomarkers Prev., 1996, 5, 587-593.

6 L. Zhang, Q. Y. Chen, H. Liu, L. Q. Tang and H. Q. Mai, Drug Des., Dev. Ther., 2013, 7, 37-52.

7 S. D. Stoker, J. N. A. van Diessen, J. P. de Boer, B. Karakullukcu, C. R. Leemans and I. B. Tan, Curr. Treat Options, 2013, 14, 475-491.

8 T. Xu, J. Tang, M. Gu, L. Liu, W. Wei and H. Yang, Curr. Oncol., 2013, 20, E406-E419.

9 E. T. Chang and H. O. Adami, Cancer Epidemiol., Biomarkers, 2006, 15, 1765-1777.

10 M. Arnold, M. A. Wildeman, O. Visser, H. E. Karim-Kos, J. M. Middeldorp, R. Fles, I. B. Tan and J. W. Coebergh, Oral Oncol., 2013, 49, 237-243.

11 A. Hildesheim and C. P. Wang, Semin. Cancer Biol., 2012, 22, 107-116.

12 D. C. Lin, X. Meng, M. Hazawa, Y. Nagata, A. M. Varela, L. Xu, Y. Sato, L. Z. Liu, L. W. Ding, A. Sharma, B. C. Goh, S. C. Lee, B. F. Petersson, F. G. Yus, P. Macary, M. Z. Oo, C. S. Ha, H. Yang, S. Ogawa, K. S. Loh and H. P. Koeffler, Nat. Genet., 2014, 46, 866-871.

13 J. H. Lee, M. E. Miele, D. J. Hicks, K. K. Phillips, J. M. Trent, B. E. Weissman and D. R. Welch, J. Natl. Cancer Inst., 1996, 88, 1731-1737.

14 T. Ohtaki, Y. Shintani, S. Honda, H. Matsumoto, A. Hori, K. Kanehashi, Y. Terao, S. Kumano, Y. Takatsu, Y. Masuda, Y. Ishibashi, T. Watanabe, M. Asada, T. Yamada, M. Suenaga, C. Kitada, S. Usuki, T. Kurokawa, H. Onda, O. Nishimura and M. Fujino, Nature, 2001, 411, 613-617.

15 M. Bilban, N. Ghaffari-Tabrizi, E. Hintermann, S. Bauer, S. Molzer, C. Zoratti, R. Malli, A. Sharabi, U. Hiden, W. Graier, M. Knofler, F. Andreae, O. Wagner, V. Quaranta and G. Desoye, J. Cell Sci., 2004, 117, 1319-1328.

16 T. A. Martin, G. Watkins and W. G. Jiang, Clin. Exp. Metastasis, 2005, 22, 503-511.
17 D. K. Dhar, H. Naora, H. Kubota, R. Maruyama, H. Yoshimura, Y. Tonomoto, M. Tachibana, T. Ono, H. Otani and N. Nagasue, Int. J. Cancer, 2004, 111, 868-872.

18 M. Ikeguchi, K. Yamaguchi and N. Kaibara, Clin. Cancer Res., 2004, 10, 1379-1383.

19 T. Masui, R. Doi, T. Mori, E. Toyoda, M. Koizumi, K. Kami, D. Ito, S. C. Peiper, J. R. Broach, S. Oishi, A. Niida, N. Fujii and M. Imamura, Biochem. Biophys. Res. Commun., 2004, 315, 85-92.

20 M. D. Ringel, E. Hardy, V. J. Bernet, H. B. Burch, F. Schuppert, K. D. Burman and M. Saji, J. Clin. Endocrinol. Metab., 2002, 87, 2399.

21 C. Yan, H. Wang and D. D. Boyd, J. Biol. Chem., 2001, 276, 1164-1172.

22 S. Peng, S. Fan, X. Li, L. Wang, H. Liu, M. Zhou, L. Wang, S. Shen and G. Li, Cancer Sci., 2007, 98, 341-349.

23 J. Ou, F. Pan, P. Geng, X. Wei, G. Xie, J. Deng, X. Pang and H. Liang, Int. J. Radiat. Oncol., Biol., Phys., 2012, 82, e685691.

24 B. E. Elliott, J. A. Meens, S. K. SenGupta, D. Louvard and M. Arpin, Breast Cancer Res., 2005, 7, R365-R373.

25 S. Chen, W. Chen, X. Zhang, S. Lin and Z. Chen, Int. J. Oncol., 2016, 48, 1391-1398.

26 D. J. Sieg, C. R. Hauck and D. D. Schlaepfer, J. Cell Sci., 1999, 112(Pt 16), 2677-2691.

27 S. T. Lim, X. L. Chen, Y. Lim, D. A. Hanson, T. T. Vo, K. Howerton, N. Larocque, S. J. Fisher, D. D. Schlaepfer and D. Ilic, Mol. Cell., 2008, 29, 9-22.

28 M. K. Wendt and W. P. Schiemann, Breast Cancer Res., 2009, 11, R68.

29 Y. Pylayeva, K. M. Gillen, W. Gerald, H. E. Beggs, L. F. Reichardt and F. G. Giancotti, J. Clin. Invest., 2009, 119, 252-266.

30 M. Luo, H. Fan, T. Nagy, H. Wei, C. Wang, S. Liu, M. S. Wicha and J. L. Guan, Cancer Res., 2009, 69, 466-474.

31 T. J. Belbin, B. Singh, R. V. Smith, N. D. Socci, V. B. Wreesmann, M. Sanchez-Carbayo, J. Masterson, S. Patel, C. Cordon-Cardo, M. B. Prystowsky and G. Childs, Arch. Otolaryngol., Head Neck Surg., 2005, 131, 10-18. 